\title{
Easy Ontology without Deflationary Metaontology
}

DANIEL Z. KORMAN

\section{University of California}

In Ontology Made Easy, Amie Thomasson defends two striking deflationary theses. The first is epistemological: there are easy ways of knowing whether numbers, properties, and other such hotly disputed entities exist. The second is metaontological: there is something wrong with prolonged debates about the existence of such objects. I am interested in the relationship between the two. As we will see, the epistemological thesis does not by itself entail the metaontological thesis. Rather, the case for deflationary metaontology rests largely on a controversial doctrine about the possible meanings of 'object'.

There is much of value in the book that I won't have space to explore. Thomasson makes a strong case that her easy approach to ontology makes her the true heir of Carnap, that this approach has been wrongly neglected in favor of quantifier variantism, and that it can be defended against a wide range of objections. Ontology Made Easy is cutting edge work in metaontology and should be required reading for anyone interested in deflationary approaches in metaphysics.

\section{Epistemology and Metaontology}

One accepts an easy approach to existence questions if one accepts (i) that "all wellformed existence questions may be answered by conceptual and/or empirical work" and (ii) that "at least some disputed existence questions may be answered by means of trivial inferences from uncontroversial premises" (128). ${ }^{1}$ I will be focusing exclusively on (ii), the claim that there are a range of existence questions that are especially easy to answer. For Thomasson, these include (but are not limited to) questions about the existence of numbers, tables, properties, propositions, and marriages, all of which she takes to be easily answerable in the affirmative.

When Thomasson says that existence questions are easily answerable, I take her to be endorsing the following thesis about what we can know:

(EA) One can come to know the answers to certain disputed existence questions on the basis of trivial inferences from uncontroversial premises. $^{2}$

Unless otherwise noted, all references are to Thomasson (2015).

See, e.g., pp. 148 and 203. She sometimes makes only the weaker claim that one is entitled to accept the answers on the basis of such inferences; see, e.g., pp. 142-143 and 232-235. 
For instance, one can come to know that there are numbers by inferring it from the uncontroversial premise that there are nine Supreme Court justices:

(A1) There are nine Supreme Court justices.

(A2) If there are nine Supreme Court justices, then there are numbers (e.g., the number of justices).

(A3) So, there are numbers.

The linking premise in the reasoning- $\mathrm{A} 2$ - is not itself uncontroversial. But (the idea goes) it is true, and, moreover, one can know that it is true without engaging in any hard philosophical work: anyone who understands the term 'number' is already thereby in a position to see straightaway that A2 is true. Thus, those who are willing to infer A3 from A1-because they are either ignorant of, or else undeterred by, the philosophical challenges to A2 - can thereby secure knowledge of A3 in this easy way.

The other key component of Thomasson's overall deflationary view is the following deflationary metaontological thesis:

(DM) Philosophical debates about existence questions are pointless.

Or, as she other times puts it, there is something "wrong" or "amiss" with the debates. ${ }^{3}$ At times, Thomasson seems to suggest that DM follows immediately from the EA. For instance:

"although on this view the disputed existence questions are meaningful and answerable (generally in the positive), they turn out to be answerable so trivially that the 'hard' debates about these issues that have so exercised metaphysicians in recent decades seem misguided and pointless"

(158-159; cf. 22, 128, and 167).

But, as I hope to show, this move from straightforward answerability to DM is far too quick.

\section{Defeating Easy Knowledge}

Things that are straightforwardly known can sometimes face legitimate challenges. Kate was at the conference last week, and Julie clearly remembers seeing her there. Julie thereby knows, in a straightforward way, that Kate was at the conference. If I then challenge her belief that Kate was at the conference with compelling (albeit misleading) evidence, then Julie's belief is defeated: she cannot rationally go on believing that Kate was at the conference unless she can somehow assure herself that my evidence is misleading. A debate about whether Kate was there - centered on the status of the evidence to the contrary - would not be pointless. The point would be to address the putative defeaters.

The same goes for easily answerable philosophical questions. As an illustration, take the free will debate. Compatibilists of a certain stripe will say that if $S$ chose to $\phi$ without being coerced or manipulated into $\phi i n g$, then $S$ freely chose to $\phi$. Suppose they're right about this. In that case, questions of freedom are easily answerable in the relevant 
sense. Lenny, who is unaware of the arguments for incompatibilism, can know that some of his choices are free simply by means of a trivial inference from the uncontroversial premise that some of his choices weren't coerced or manipulated:

(B1) I chose to order dessert without being coerced or manipulated.

(B2) If so, then I freely chose to order dessert.

(B3) So, I freely chose to order dessert.

B2 of course is not uncontroversial, but then neither is A2. What makes this a trivial inference is that one doesn't need any philosophical sophistication in order to know that B2 is true; it seems to be exactly on a par with A2.

Still, the debate between compatibilists and incompatibilists is perfectly sensible. Why? Because the incompatibilist's arguments give us serious (albeit, we are supposing, misleading) reason to think that even choices that are not coerced or manipulated are unfree; more is required for freedom. Yes, B3 is easily known if true, but that doesn't mean its truthand a fortiori its knowability — can't legitimately be called into question. Any entitlement we have to believe that our choices are free on the basis of such trivial inferences is surely going to be defeasible, and the status of putative defeaters can legitimately be debated.

Thomasson will presumably agree that questions about free choice can legitimately be debated despite being easily answerable. But she will likely insist that there is a crucial disanalogy between these questions and the indicated existence questions. In the former but not the latter, she will say, there is a sensible debate to be had about the application conditions of the relevant terms. The free will incompatibilist is able to provide a sensible account of what more is required for the term 'free' to apply to a choice, for instance that the choice not be determined by any factors outside one's control. Can eliminativists about numbersor, for that matter, realists in search of a question worthy of debate-likewise offer a sensible alternative to A2 to serve as application conditions for 'number'?

Thomasson thinks that the best eliminativists can do is to insist that A2 be replaced with something like A2*:

(A2*) If there are nine Supreme Court justices, and there is an object that numbers the justices, then there are numbers.

In slogan form, they can "objectify" the application conditions, by explicitly requiring the existence of some relevant object. ${ }^{4}$ Thomasson maintains, however, that there cannot be any sensible debate between realists and eliminativists about whether such objectified application conditions are satisfied. And if she's right about that, then it would seem that $\mathrm{DM}$ is true: there really is nothing sensible for realists and eliminativists to be debating.

\section{The Meanings of 'Object'}

Why think there can't be sensible debate about objectified application conditions like A2*? Thomasson argues that friends of the objectified application conditions face a dilemma (108-111). There are two ways of understanding 'object', she says, neither of which will serve the eliminativist's purposes. First, there is the sortal use, whose application conditions are roughly as follows: there is an S-object (' $\mathrm{S}$ ' for sortal) in some

See, e.g., pp. 108-109, 195, and 292. 
situation if there is a medium-sized region of space in that situation whose contents are well bonded together and are independently mobile from surrounding stuff. ${ }^{5}$ In short, Sobjects are medium-sized dry goods, or "Spelke objects". Second, there is the covering use, whose application conditions are roughly as follows: there is a C-object (' $\mathrm{C}$ ' for covering) in some situation if we have some sortal concept whose application conditions are satisfied in that situation.

Here is the rub. If 'object' is read as $S$-object, then A2* can't be a sensible target for debate since all sides agree that numbers aren't medium-sized dry goods. If 'object' is read as $C$-object, then A2* is no more demanding than A2, since the application conditions for one of our sortal terms, 'number', are satisfied so long as there are nine justices. ${ }^{6}$ And since these are the only intelligible ways of understanding 'object' in A2*, the idea goes, there is no way of understanding the proposed objectified application conditions that can deliver a sensible subject of debate.

The argument from the possible uses of 'object' to metaontological deflationism about debates over the existence of numbers can then be framed as follows:

(C1) There is something wrong with philosophical debates about the existence of numbers unless there are objectified application conditions for 'number' that are (i) well formed and (ii) such that disputants disagree about whether they are satisfied.

(C2) If 'object' means $S$-object or C-object in objectified application conditions for 'number', then disputants do not disagree about whether they are satisfied.

(C3) If 'object' does not mean S-object or C-object in objectified application conditions for 'number', then the objectified application conditions are ill formed.

(C4) So, there is something wrong with philosophical debates about the existence of numbers.

Before I turn to assessing the argument, it is worth noting just how much work is being done in the book by Thomasson's contention that there are only these two readings of 'object'. It plays a key role in:

(i) her argument that Carnap is not a quantifier variantist (73 n.29),

(ii) her argument that fictionalists cannot explain what it takes for a sentence like A3 to be literally true (195-197),

(iii) her defense of arbitrary mereological fusions and a plenitudinous ontology of material objects $(214,221)^{7}$

6 One might worry that questions are being begged here, insofar as Thomasson is assuming that the application conditions for 'number' are non-objectified. I won't pursue this worry here.

7 The S-object/C-object dilemma doesn't explicitly come up here, but it must be playing a role in the background. Thomasson says that her view delivers extraordinary material objects like gollyswoggles and trout-turkeys. Why is that? The idea, I take it, is that 'trout-turkey' was introduced with the following application conditions: there is a trout-turkey if there is a front half of a trout, a back half of a turkey, and an object composed of the two. But (the idea goes) there trivially will be such a C-object and it's irrelevant that it isn't an S-object. 
(iv) her response to the objection that her simple realism requires that there "magically" be enough objects to render all the disputed existence claims true (218220), and

(v) her argument that there is no way to make sense of an alleged "heavyweight" reading of the quantifiers in A3 (292-293).

So there is quite a bit riding on her claim that 'object' must mean either S-object or $C$ object. $^{8}$

I deny C3. There is a further sense of 'object' - call it U-object, for 'unrestricted'which we both can and do understand, one that applies unrestrictedly to everything, to all things, to whatever is in the largest domain, that applies to something no matter what it is. ${ }^{9}$ Thomasson will surely object that the dilemma arises anew for every one of these characterizations: 'thing' — in 'all things', 'something', 'the largest domain [of things]'likewise has only a sortal and covering reading. ${ }^{10}$ Which of course I will deny, insisting that there is a well-understood, unrestricted reading of 'thing' as well. Which leaves us at something of an impasse.

In what follows, I try to move the debate forward by challenging what I see as Thomasson's main argument in defense of C3 (\$4) and by offering some reasons of my own for rejecting C3 (§5).

\section{The Case Against the Unrestricted Reading of 'Object'}

When Thomasson does explicitly entertain the possibility that 'object' is being used to mean something other than S-object or C-object, her complaint is that it must in those cases lack application conditions (219, 292-293). Importantly, for Thomasson, in order for a term to count as having application conditions, in the intended sense, there must be something like rules that speakers with no antecedent understanding of the term can follow to determine whether the term applies in a given situation (89-90). In short, application conditions must be nontrivial; "“U-object' applies iff there is a U-object" won't cut it.

The argument for C3 can then be stated as follows.

(D1) If 'object' is not being used to mean S-object or C-object, then it is being used without nontrivial application conditions.

(D2) If 'object' is being used without nontrivial application conditions, then claims in which it appears are ill formed.

(C3) So, if 'object' is not being used to mean S-object or C-object in objectified application conditions for 'number', then the objectified application conditions are ill formed.

As Schaffer observes (2009: 152), it also bears much of the argumentative weight in her (2007).

I say that it is a "further" sense, but actually I doubt that 'object' ever means C-object or S-object. My own view is that it only ever means U-object or material continuant. But I won't have the space to defend that view here.

And see her (2009: §6) against characterizing the additional reading of 'object' using quantifiers and the identity predicate. 
Let's just grant D1, that 'object' lacks nontrivial application conditions when we try to use it to mean $U$-object. ${ }^{11}$ Why accept D2? Some meaningful and intelligible expressions very plausibly have no nontrivial application conditions, for instance logical expressions like 'and', 'or', and 'if'. So why think 'object' (or 'thing' or 'something') is meaningful only if it is used with nontrivial application conditions?

Here is what Thomasson has to say on the matter: "if a term lacks application conditions, we cannot (in the object language) evaluate claims about whether or not the corresponding entities exist" (219). But what does it mean to say that such a claim is unevaluable? Perhaps the idea is that 'numbers exist' would be semantically unevaluable (i.e., incapable of having a truth value) because the application conditions for 'number' given in A2* - that there be an object that numbers the justices - are ill formed. But then what we have is just a reiteration of D2, not an argument for it. Alternatively, perhaps the idea is that 'numbers exist' would then be epistemically unevaluable, since, without nontrivial application conditions for 'object' to work with, we cannot understand A2* well enough to assess whether the antecedent is satisfied. But this seems just to beg the question against the envisaged friends of U-objects, who challenge D2 on the grounds that 'object' is among the expressions that are meaningful and intelligible despite lacking nontrivial application conditions.

So, absent further argument, I am inclined to reject D2 and C3, thus clearing the way for denying DM. ${ }^{12}$

\section{In Defense of Another Sense}

I've argued that Thomasson fails to establish C3. But is there any good reason to deny it? Is there reason to think that there is another available reading of 'object' - one that applies to everything, even if it isn't a medium-sized dry good, even if it doesn't fall under any of our sortal concepts? To be honest, it just seems obvious to me that there is such a reading. When Thomasson tells us that there is no intelligible notion there, and that U-object is just some philosopher's failed invention, I am reminded of Kripke's reaction to Quine's anti-essentialism. Quine claimed that essential properties are made up by some confused philosopher and that there is no sense to be made of the claim that Nixon himself (however you what to describe him) has the property of possibly losing the election. To which Kripke replied: "now which one is being the philosopher here?"13

Of course, what's obvious to me won't be obvious to everyone. So, in hopes of moving the debate forward, let me give two arguments that 'object' sometimes means $U$-object. ${ }^{14}$

Here is the first argument, which is meant to show that, given her other commitments, Thomasson herself ought to recognize a further sense of 'object'. Consider the following sentence:

(H) There are abstract objects for which no one has a sortal concept. ${ }^{15}$

11

Though see Schaffer (2009: 150-151) for some candidate application conditions.

One can perhaps extrapolate an additional argument for D2 from Thomasson's discussion of the qua problem (95). See my (2015: §4.4.2) for discussion of an argument from the qua problem as it arises in her (2007).

Kripke (1980: 41).

See also Evnine (forthcoming: $160 \mathrm{n} .17$ ) for the makings of an argument that something like U-object may be playing an implicit role in Thomasson's own thinking about "co-application conditions".

' $\mathrm{H}$ ' for Hamlet: "there are more things in heaven and earth ... than are dreamt of in your philosophy." 
Thomasson herself will presumably want to affirm $(\mathrm{H})$. She defends a simple realism according to which numbers, propositions, and other such entities are mind-independent existents that long pre-dated us (chapter 3.3). And presumably she'd agree that there is more out there to be discovered, that we have yet to name or conceptualize. Indeed, $(\mathrm{H})$ should strike her as trivially true, since by her own lights all we have to do is cook up some new sortal term and application conditions (meeting fairly undemanding constraints), ${ }^{16}$ and we can be confident that there are entities answering to the term. And since, by the lights of her simple realism, the cooking doesn't itself bring these entities into existence, they must already be out there.

Yet reading 'object' as $S$-object turns $(\mathrm{H})$ into the absurd claim that there are abstract medium-sized dry goods; and reading it as C-object turns $(\mathrm{H})$ into a trivial falsehood, true iff abstracta belonging to sortal concepts we have don't belong to sortal concepts we have. To find a true reading of $(\mathrm{H})$, Thomasson must allow that there is some other reading of 'object'. Perhaps U-object.

Here is a second argument. There has been some debate over whether 'the Supreme Court' is referentially singular or referentially plural. ${ }^{17}$ Does it refer plurally just to the nine justices, or does it refer singularly to a single object composed of the justices? In other words, is the following sentence true?

(S) The Supreme Court is one object.

This is a sensible question with no obvious answer. But the question cannot be whether the Supreme Court is an S-object, since it plainly isn't: whatever it is, it's not a mediumsized dry good whose parts are "well bonded". Nor can the question be whether the Supreme Court is a C-object. Sentences of the form o is one C-object come out true so long as, for some kind $\mathrm{K}$ for which we have a sortal concept, $o$ is one $K$ comes out true. And, since trivially the Supreme Court is a supreme court, it follows that the Supreme Court is one C-object. ${ }^{18}$ So there is no way to make sense of the sensible debate over (S) unless we recognize some other sense of 'object'. Perhaps $U$-object.

One may object that this argument is question-begging: Thomasson has already denied that there can be sensible debates about existence questions, so why should she grant that there can be sensible debate about (S)? Notice, though, that (S) is something even a fairly permissive ontologist can puzzle over. Even supposing that there is some object composed of the justices, there is still the question of whether 'the Supreme Court' picks out that object or rather refers plurally to the justices themselves. Accordingly, the debate over (S) needn't be an "existence debate": even those who are completely in agreement about what there is can disagree about what the Supreme Court is. It is therefore entirely

\footnotetext{
16 See her chapter 8.2 for discussion of the constraints on successfully introducing new referring expressions.

17 See, e.g., Uzquiano (2004), López de Sa (2007), Ritchie (2013: §2), and Korman (2015: §8.3.2).

18 Can $I$ agree that the Supreme Court is a supreme court without thereby conceding that the Supreme Court is a (one) U-object? Yes. Cf. McKay (2006: 42) on 'a dozen': "There is no single thing that is a dozen bagels, there are twelve things that are a dozen bagels... The phrase 'a dozen' is only misleadingly singular."
} 
open to proponents of DM to accept that this is a sensible dispute. No questions are begged.

\section{Conclusion}

I have been criticizing Thomasson's defense of her deflationary metaontological thesis, which as we saw ultimately rests on the claim that there is no sense to be made of the metaphysician's unrestricted use of 'object'. In doing so, I hope to have cleared the way for my preferred view of these matters. EA is true: absent defeaters, the trivial inferences are indeed a route to knowing the very things that realists affirm and eliminativists deny. But DM is false: prolonged debates about existence questions do have a point. The point is to assess strategies for reinstating or circumventing easy routes to knowledge once putative defeaters (in the form of arguments for eliminativism) have rendered them suspect. $^{19}$

\section{References}

Evnine, Simon (forthcoming), 'Much Ado About Something-From-Nothing: Problems for Ontological Minimalism', in Stephan Blatti and Sandra Lapointe (eds.), Ontology After Carnap (Oxford: Oxford University Press).

Korman, Daniel Z. (2015), Objects: Nothing Out of the Ordinary (Oxford: Oxford University Press).

Kripke, Saul (1980), Naming and Necessity (Cambridge: Harvard).

López de Sa, Dan (2007), 'The Chief Supreme Court Justice: A Metaphysical Puzzle?', Crítica 39: 61-68.

McKay, Thomas J. (2006), Plural Predication (Oxford: Oxford University Press).

Ritchie, Katherine (2013), 'What are Groups?', Philosophical Studies 166: 257-272.

Schaffer, Jonathan (2009), 'The Deflationary Metaontology of Thomasson's Ordinary Objects', Philosophical Books 50: 142-157.

Thomasson, Amie (2007), Ordinary Objects (Oxford: Oxford University Press).

_ (2009), 'Answerable and Unanswerable Questions', in David Chalmers, David Manley, and Ryan Wasserman (eds.), Metametaphysics (Oxford: Oxford University Press), pp. 444-471. (2015), Ontology Made Easy (Oxford: Oxford University Press).

Uzquiano, Gabriel (2004), 'The Supreme Court and the Supreme Court Justices: A Metaphysical Puzzle', Nô̂s 38: 135-153.

Thanks to Chad Carmichael, Simon Evnine, David Kovacs, David Palmer, and Amie Thomasson for helpful feedback. 